This item was submitted to Loughborough's Research Repository by the author.

Items in Figshare are protected by copyright, with all rights reserved, unless otherwise indicated.

\title{
The control of non-twisting somersaults using configuration changes
}

PLEASE CITE THE PUBLISHED VERSION

http://dx.doi.org/10.1016/0021-9290(96)00034-6

\section{PUBLISHER}

Pergamon (๑ Elsevier Science Ltd.)

\section{VERSION}

AM (Accepted Manuscript)

\section{LICENCE}

CC BY-NC-ND 4.0

\section{REPOSITORY RECORD}

Yeadon, Maurice R., and E.C. Mikulcik. 2019. "The Control of Non-twisting Somersaults Using Configuration Changes”. figshare. https://hdl.handle.net/2134/11528. 
This item was submitted to Loughborough's Institutional Repository (https://dspace.lboro.ac.uk/) by the author and is made available under the following Creative Commons Licence conditions.

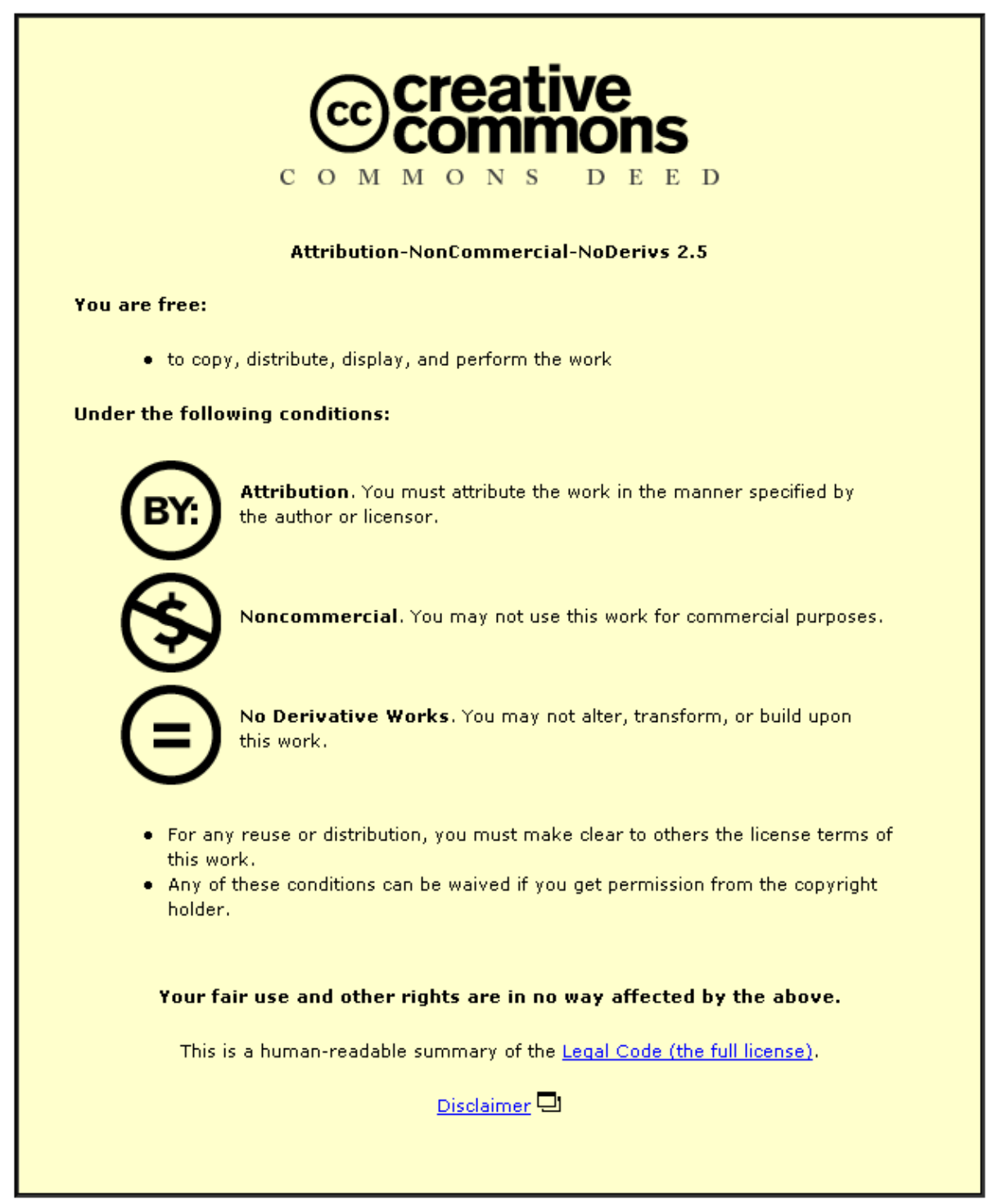

For the full text of this licence, please go to: http://creativecommons.org/licenses/by-nc-nd/2.5/ 


\title{
THE CONTROL OF NON-TWISTING SOMERSAULTS USING CONFIGURATION CHANGES
}

\author{
M. R. Yeadon*† and E. C. Mikulcik $\ddagger$ \\ *Department of Sports Science,Loughborough University, U.K.; \\ $\dagger$ Faculty of Physical Education, University of Calgary; and \\ $\ddagger$ Department of Mechanical Engineering, University of Calgary, Canada
}

\begin{abstract}
Theoretical analyses have shown that rotations of a rigid body about the principal axis corresponding to the intermediate principal moment of inertia are unstable. This poses a potential problem for gymnasts who perform double somersaults without twist in a layout configuration. A computer simulation model is used to investigate configurational strategies for controlling such movements. It is shown that the build up of twist is not reduced by abduction of the arms but can be controlled by adopting a configuration with sufficient body flexion. For somersaults with a straight body, control in the form of asymmetrical arm abduction accelerations, based upon twist angular velocity and angular acceleration, is capable of preventing a build up of twist providing that the feedback time delay is less than a quarter somersault.
\end{abstract}

Keywords: Control; Simulation; Somersault; Stability; Twisting.

\section{INTRODUCTION}

In aerial sports such as diving, trampolining and gymnastics the basic movements comprise somersaults with and without twist. For successful execution of a twisting somersault the athlete must ensure that the required number of twists are completed for a non-twisting somersault and that no twist is seen occur. Theoretical analyses have shown that a rigid body is stable for rotations about the principal axes corresponding to maximum and minimum moments of inertia but unstable for rotations about the principal axis corresponding to the intermediate moment of inertia (Hinrichs, 1978; Marion, 1965). This instability arises because rigid body motions fall into two general modes centred on motions about the maximum and minimum principal axes and appear as either wobbling somersaults or twisting somersaults (Yeadon,1993a). Rotations about the intermediate principal axis lie near the boundary separating the two different modes of motion (Yeadon, 1993a). For example, a piked (hips flexed) somersault about the lateral axis, corresponding maximum to moment of inertia, will appear to be a pure somersault since the angular velocity vector will remain close to this principal axis throughout the movement. In the case of a layout (nominally straight) somersault about the lateral axis, corresponding to the intermediate principal moment of inertia, any slight deviation of the angular velocity vector from this principal axis will eventually lead to a substantial build up of twist sufficient to change backwards rotation into forwards rotation (Yeadon, 1993a).

Nigg (1974) suggested that the arms could be extended laterally in a layout somersault order in to minimise the influence of the instability. While it might be true that extending the arms will reduce the twist rate, the problem of instability still remains and the proposed strategy at best can only delay the inevitable build up of twist. If the build up of appreciable twist does not occur until after the completion of two somersaults then instability would not present a practical problem for a gymnast. If the build up of twist becomes noticeable after one somersault then instability would only present a problem for multiple somersaults. If the build up of twist is noticeable before the completion of one somersault then instability would pose a problem in all non-twisting straight somersaults. Thus it is of great relevance to determine the amount of somersault at which the twist becomes apparent.

Hinrichs(1978) determined the directions of the principal axes during tucked, piked and layout somersaults from a trampoline. In the tucked and piked somersaults the principal axis corresponding to 
maximum moment of inertia remained close to the angular momentum vector, while for the layout somersault the intermediate principal axis remained close to the angular momentum vector. This apparent stability about the intermediate axis lead Hinrichs to speculate that the trampolinist must have made adjustments during flight to prevent the build up of twist. On the other hand, it could be argued that, although a somersault in the straight position is theoretically unstable, in practice the body is not straight at the beginning and end of the movement so that the instability has insufficient time to produce noticeable twist in a single somersault. It would be of interest to determine, therefore, the extent to which flexion of the hips and spine can delay the build up of twist. If neither of the strategies suggested (arm abduction and body flexion) is capable of limiting the effects of the instability, the remaining possibility is that in-flight corrections are made to reverse any build up of twist.

In a twisting somersault asymmetrical arm movements may be used to alter the tilt angle so that the twist is stopped or even reversed (Yeadon, 1993b). It should be possible to ensure that the twist angle remains small during an unstable straight somersault by making corrective arm movements. In a backward somersault abduction of the left arm will produce a twist to the right whilst abduction of the right arm will produce a twist to the left.

In this study three alternative strategies for controlling non-twisting somersaults using changes of body configuration are investigated. The three strategies comprise (a) arm abduction, (b) flexion of the hips and spine, and (c) asymmetrical arm adduction/abduction.

\section{METHODS}

In order to investigate these strategies for controlling the build up of twist in layout somersaults, a computer simulation model of aerial movement was used (Yeadon et al., 1990). This model comprised 11 segments representing chest and head, thorax, pelvis, upper arms, lower arms, upper legs and lower legs. Inertia parameters were calculated from anthropometric measurements of an elite trampolinist using a mathematical inertia model of the human body (Yeadon, 1990b). Input to the model comprised: (a) initial conditions in the form of the components of angular momentum about the mass centre and initial values of three angles defining body orientation together with (b) time histories of 14 angles defining body configuration. Output of the model comprised the time histories of the angles of somersault, tilt and twist which define the orientation of the body in space. Hypothetical simulations were run in order to determine the outcomes of the three proposed methods of control.

To determine the effect of arm abduction for somersaults in a straight body configuration, three simulations were first carried out with the arms adducted close to the body. Sufficient angular momentum was used to produce a straight double somersault. A small perturbation was introduced into each simulation by specifying an initial asymmetry in arm abduction angles. These asymmetries had magnitudes $0.1^{\circ}$, $1^{\circ}$ and $10^{\circ}$. Subsequently, three more simulations were carried out in which each arm was approximately perpendicular to the mid-line of the trunk. Initial arm asymmetries of $0.1^{\circ}, 1^{\circ}$ and $10^{\circ}$ were again used to perturb the motion. A rigid configuration was maintained throughout each simulation.

To determine the effect of flexion of the hips and spine on the stability of layout somersaults, simulations were carried out in which the flexion angle between upper trunk and thighs remained constant. Flexion angles ranged from $120^{\circ}$ to $180^{\circ}$ which corresponded to a straight body configuration. Perturbations were introduced using initial arm asymmetries of $0.1^{\circ}, 1^{\circ}$ and $10^{\circ}$ and a fixed configuration was maintained throughout each simulation. The results of these simulations established the extent to which flexion of the body could limit the build up of twist during a double somersault.

To evaluate the capabilities of asymmetrical arm abduction for correcting the build up of twist, the equations of motion of a model, comprising two arms and one body segment, were first linearised by assuming that perturbations remained small. This permitted an analytical consideration of the prospective control strategies for arbitrarily small perturbations. The successful control strategy indicated by this analysis was then incorporated into the computer simulation model in order to determine whether this strategy would also provide control in a system that was non-linear and for perturbations that were not arbitrarily small.

The equations of motion for an asymmetrical arm abduction controller may be derived as follows. Suppose that abduction of one arm is accompanied by adduction of the other so that the sum $\varepsilon_{a}+\varepsilon_{b}$ of the abduction angles of the left and right arms from the mid-line of the trunk remains constant. Let $\dot{\varepsilon}=-\dot{\varepsilon_{a}}=\dot{\varepsilon_{b}}$ be the rate of change of arm abduction angles. As shown in Yeadon (1990c) the equation 
of motion will be:

$$
\boldsymbol{h}=\boldsymbol{I} \omega+\boldsymbol{h}_{r e l}
$$

where $\boldsymbol{h}$ is the total angular momentumabout the mass centre, $\boldsymbol{I}$ the whole body inertia tensor about the mass centre, $\omega$ the angular velocity of the system, and $\boldsymbol{h}_{r e l}$ the angular momentum corresponding to internal movements. The equation may be written as:

$$
\left[\begin{array}{c}
h \cos \theta \cos \psi \\
-h \cos \theta \cos \psi \\
h \sin \theta
\end{array}\right]=\left[\begin{array}{ccc}
B & 0 & 0 \\
0 & A & 0 \\
0 & 0 & C
\end{array}\right] \times\left[\begin{array}{c}
\dot{\phi} \cos \theta \cos \psi+\dot{\theta} \sin \psi \\
-\dot{\phi} \cos \theta \sin \psi+\dot{\theta} \cos \psi \\
\dot{\phi} \sin \theta+\dot{\psi}
\end{array}\right]+\left[\begin{array}{c}
0 \\
I_{a} \dot{\varepsilon} \\
0
\end{array}\right]
$$

where $A>B>C$ are the principal moments inertia of of $\boldsymbol{I}, I_{a} \dot{\varepsilon}$ is the angular momentum associated with the arm movement and $\phi, \theta$ and $\psi$ are Cardan angles for somersault, tilt and twist corresponding to successive rotations about lateral, frontal and longitudinal axes. Equation (2) gives rise to:

$$
\begin{gathered}
h=B \dot{\phi}+B \dot{\theta} \sec \theta \tan \psi \\
-h \sin \psi=-A \dot{\phi} \sin \psi+A \dot{\theta} \sec \theta \cos \psi+I_{a} \dot{\varepsilon} \sec \theta \\
h \sin \theta=C \dot{\phi} \sin \theta+C \dot{\psi}
\end{gathered}
$$

Eliminating $\dot{\phi}$ from (3) and (4) gives:

$$
h(A-B) \sin \psi=A B \dot{\theta}\left(\sec \theta \cos \psi+\sec \theta \sin ^{2} \psi \sec \psi\right)+I_{a} B \dot{\varepsilon} \sec \theta
$$

Eliminating $\dot{\phi}$ from (3) and (5) gives:

$$
B C \dot{\psi}=h(B-C) \sin \theta+B C \dot{\theta} \tan \theta \tan \psi
$$

If the tilt and twist angles $\theta$ and $\phi$ are assumed be to small and the controlling angular velocity $\dot{\varepsilon}$ is small then (6) and (7) imply that $\dot{\theta}$ and $\dot{\psi}$ will also be small. The approximations $\sin \theta=\theta, \cos \theta=1$, $\sin \psi=\psi, \cos \psi=1$ are used in equations (61) and (7) and small quantities of the third order are neglected. Equation (6) becomes:

$$
h(A-B) \psi=A B \dot{\theta}+I_{a} B \dot{\varepsilon}
$$

Equation (7) becomes:

$$
B C \dot{\psi}=h(B-C) \theta
$$

Differentiating equation(9) and using equation(8) gives:

$$
\ddot{\psi}=k^{2} \psi-m \dot{\varepsilon}
$$

where $k^{2}=h^{2}(B-C)(A-B) / A B^{2} C$ and $m=h I_{a}(B-C) / A B C$.

A value for $I_{a} / A$ was obtained using a simulation under conditions of zero angular momentum with the model of Yeadon et al. (1990). The arms were moved through a small angle $\varepsilon$ and the change in the tilt angle of the longitudinal principal axis was noted. When $h=0$, equation (8) gives $I_{a} / A=\dot{\theta} / \dot{\varepsilon}$ and so $\theta / \varepsilon$ was used as an approximation to $I_{a} / A$.

In equation(10) $\psi$ is the output variable, which should remain small if the build up of twist is to be prevented, and $\dot{\varepsilon}$ is the control variable. The transfer function, obtained by taking Laplace transforms and assuming zero initial conditions is:

$$
\left(s^{2}-k^{2}\right) \psi(s)=-m \dot{\varepsilon}(s)
$$

or

$$
\frac{\psi(s)}{\dot{\varepsilon}(s)}=\frac{-m}{s^{2}-k^{2}}
$$


(a)

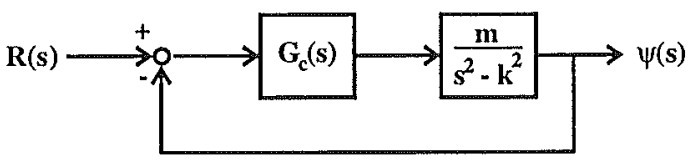

(b)

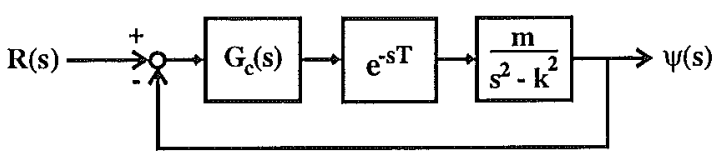

Figure 1: Structure closed-loop for control with (a) zero time delay and (b) finite time delay.

where $s$ is the complex variable $\sigma+j \omega$.

With closed-loop control, the structure can be drawn in standard control systems form as shown in Fig. 1(a) where $G_{c}(s)$ is the controller's transfer function and $R(s)$ is the desired value of the output $\psi(s)$ which in this case is zero. When there is a time delay $T$ in the system, it can be incorporated using the translation property of Laplace transforms as shown in Fig. T(b).

The characteristic equation of this system is $1+G(s)=0$ where

$$
G(s)=\frac{G_{c}(s) m e^{-s T}}{s^{2}-k^{2}}
$$

For stability, all roots of the characteristic equation (in s) must have negative real parts. When there is a time delay, the characteristic equation is not a polynomial so the solution is not straightforward. However, the stability of the system can be determined by investigating whether there are any roots with positive real parts using the Nyquist Stability Criterion (Van de Vegte, 1986). The procedure is to map the Nyquist contour, which encloses the entire right-hand side of the complex $s$-plane, into the $G(s)$ plane in a clockwise direction to form the Nyquist diagram. The number of zeros of the characteristic equation having positive real parts is $Z=N+P$ where $N$ is the number of clockwise encirclements $(-1,0 j)$ by the Nyquist diagram in the $G(s)$ plane and $P$ is the number of poles of $G(s)$ contained inside the Nyquist contour in the $s$-plane. For stability $Z$ must equal zero.

The use of proportional plus integral plus derivative (PID) controllers for stable control was investigated in order to determine which strategies are available to gymnasts.

The above analytical treatment assumes that the perturbations of the system are arbitrarily small. In reality we may have arm symmetries where the arm abduction angles differ by asmuch as $10^{\circ}$ and the control system must be able to cope with such disturbances for the duration of a double somersault. On the other hand, stable control may not be necessary since the duration of the movement is limited. Proportional plus derivative control was incorporated into the 11 segment computer simulation model of Yeadon et al. (1990) by making the arm abduction angles change from $\varepsilon_{a}$ to $\varepsilon_{a}-\delta \varepsilon$ and from $\varepsilon_{b}$ to $\varepsilon_{b}+\delta \varepsilon$ over a time interval $0.01 T_{f}$ where $T_{f}$ is the flight time and $\delta \varepsilon=\left(K_{p} \psi+K_{d} \dot{\psi}\right) T_{f} / 100$. A time delay was also introduced by basing the correction $\delta \varepsilon$ upon earlier values of $\psi$ and $\dot{\psi}$ in a simulation.

Simulations of straight double somersaults were run for initial arm asymmetries of $0.1^{\circ}, 1^{\circ}$ and $10^{\circ}$ and suitable values of the proportional and derivative constants were found for which the build up of twist was controlled. The feedback time delay in the control loop was increased to determine the maximum delay for which control was possible and to obtain the corresponding optimum values for the control parameters. These values were then compared with the corresponding results from the theoretical analysis.

In order to address question as to whether in-flight the corrections are actually used, a double layout somersault performed by an elite trampolinist was filmed using two $16 \mathrm{~mm}$ cameras operating at 70 frames per second. The locations of both cameras and two calibration points were surveyed and threedimensional reconstruction was effected using the method of Yeadon (1989). In each frame of the flight 
Table 1: Twist after one and two somersaults as a function of arm asymmetry

\begin{tabular}{lccc}
\hline Somersault & Arm asymmetry & Arms adducted & Arms abducted \\
\hline 1 & $0.1^{\circ}$ & 0.00 & 0.01 \\
1 & $1^{\circ}$ & 0.02 & 0.07 \\
1 & $10^{\circ}$ & 0.23 & 0.35 \\
2 & $0.1^{\circ}$ & 0.22 & 0.41 \\
2 & $1^{\circ}$ & 0.46 & 0.49 \\
2 & $10^{\circ}$ & 0.50 & 0.52 \\
\hline
\end{tabular}

Note: Somersault and twist values are in revolutions.

phase the wrist, elbow, shoulder, hip, knee and ankle centres were digitised for each camera view. Orientation and configuration angles were determined (Yeadon, 1990a) and segmental inertia parameter values were calculated from anthropometric measurements (Yeadon, 1990b).

The orientation of the intermediate principal axis relative to the angular momentum vector was calculated throughout each movement in order to determine whether there was sufficient flexion of the body to remove the instability. The film values of the body configuration angles and the initial orientation angles and angular momentum were used as input to the simulation model. This was done in order to establish whether a build up of twist became noticeable during the simulation. If substantial twist occurred in the simulation this would indicate that the movement was unstable and that the gymnast must have made adjustments during flight. For this movement additional simulations were carried out in which the arm abduction angles were modified from the film values using a control strategy. It is to be expected that if the simulation model is a close approximation to reality, there exist configuration histories close to those obtained from film for which there is no appreciable build up of twist in the simulation.

An experiment was conducted in order to determine whether visual feedback was necessary for the control strategy used by a gymnast. A trampolinist performed six double layout somersaults, each from a plain jump. He was then instructed to close his eyes immediately after takeoff and to open them when instructed later in the flight phase. The instruction to open the eyes was given after the completion of one and a half somersaults. This "blind" double layout somersault was attempted six times. Informed consent was obtained prior to the experiment.

\section{RESULTS}

\section{Arm abduction}

The introduction of small perturbations into simulations of straight double somersaults resulted in substantial amounts of twist even when the arms were abducted. The twist after one somersault was small when the initial arm symmetry was $0.1^{\circ}$ or $1.0^{\circ}$ but was large for an asymmetry angle of $10^{\circ}$ (Table 1). After two somersaults the twist was large in each of the six simulations. Using the abducted arm configuration did not result in reduced twist. On the contrary the instability was more evident when the arms were wide. For an initial arm asymmetry of $1^{\circ}$ the movement appeared to be a somersault with little twist followed by a somersault with a half twist (Fig. 2). Although separate simulations were carried out for rigid body configurations with arms adducted or abducted, the arms may be considered to have been instantaneously abducted immediately after takeoff.

\section{Body flexion}

The adoption of a flexed body configuration enabled the effects of instability to be reduced and even eliminated. Adopting a flexion angle of $132^{\circ}$ between upper trunk and thighs resulted in stable wobbling somersaults with little twist when the initial arm asymmetry was $0.1^{\circ}$ or $1.0^{\circ}$. For an initial arm asymmetry of $10^{\circ}$ a flexion angle of $129^{\circ}$ was required in order for the motion to follow the stable 


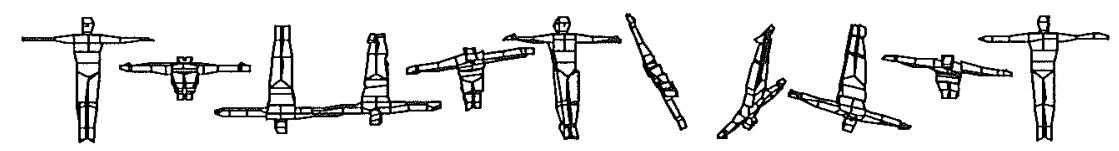

Figure 2: A rigid configuration with only $1^{\circ}$ of asymmetry in arm abduction angles produces almost a half twist after two somersaults.

Table 2: Twist after two somersaults as a function of body flexion and arm asymmetry

\begin{tabular}{lccc}
\hline \multirow{3}{*}{ Body flexion } & $0.1^{\circ}$ & $1^{\circ}$ & $10^{\circ}$ \\
& & & \\
$120^{\circ}$ & $0.00 \mathrm{~W}$ & $0.00 \mathrm{~W}$ & $0.02 \mathrm{~W}$ \\
$130^{\circ}$ & $0.00 \mathrm{~W}$ & $0.01 \mathrm{~W}$ & $0.16 \mathrm{~T}$ \\
$140^{\circ}$ & $0.01 \mathrm{~T}$ & $0.07 \mathrm{~T}$ & $0.41 \mathrm{~T}$ \\
$150^{\circ}$ & $0.03 \mathrm{~T}$ & $0.27 \mathrm{~T}$ & $0.48 \mathrm{~T}$ \\
$160^{\circ}$ & $0.11 \mathrm{~T}$ & $0.42 \mathrm{~T}$ & $0.49 \mathrm{~T}$ \\
$170^{\circ}$ & $0.22 \mathrm{~T}$ & $0.46 \mathrm{~T}$ & $0.50 \mathrm{~T}$ \\
$180^{\circ}$ & $0.22 \mathrm{~T}$ & $0.47 \mathrm{~T}$ & $0.50 \mathrm{~T}$ \\
\hline
\end{tabular}

Notes: Twist values are in revolutions; W: wobbling somersault, T: twisting somersault.

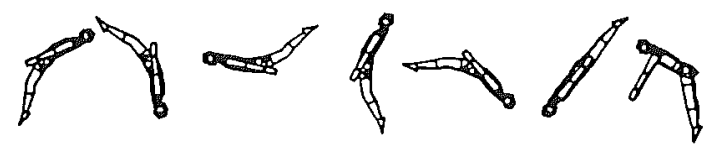

Figure 3: A double backward somersault with sufficient flexion to limit the build up of twist. 
wobbling somersault mode. Although in this case the motion was in a technically stable mode, the twist angle oscillated slowly with an amplitude of 0.24 revolutions and reached 0.14 revolutions after two somersaults. From Table 2 it can be seen that flexion provides a progressive improvement in controlling the build up of twist.

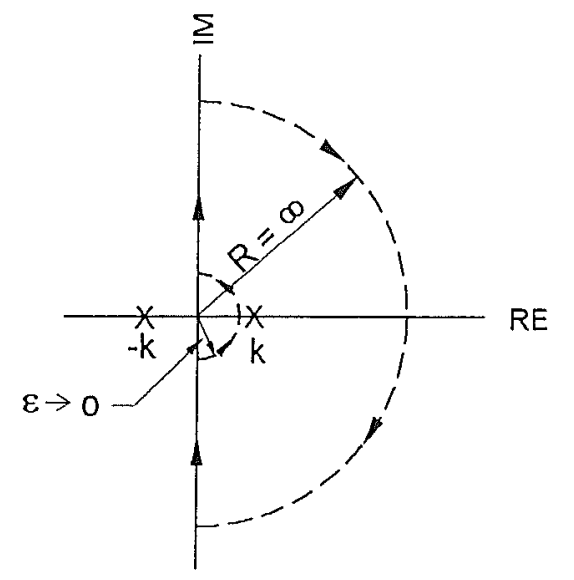

Figure 4: Nyquist contour for PID control.

In the simulation shown in Fig. 3 the abduction angle of the left arm is $1^{\circ}$ more than that of the right arm so that the principal axes are tilted through a small angle. During the first one and a half somersaults there is a body flexion angle of $145^{\circ}$ and a knee angle of $160^{\circ}$. This phase of the motion is unstable but the build up of twist is slow since the two large principal moments of inertia are approximately equal. In the last half somersault the body moves through the straight position into a stable piked somersault with a body flexion angle of $130^{\circ}$. Thus it is possible to perform an open double back somersault without appreciable build up of twist providing the flexion is not less than that shown in Fig. 3.

\section{PID control}

The investigation of PID controllers revealed that proportional plus derivative control was necessary and sufficient to provide stable operation for non-zero time delays. The controller's transfer function $G_{c}(s)=K_{p}+K_{d} s+K_{i} / s$ where $K_{p}, K_{i}$ and $K_{d}$ are the constant controller parameters so that:

$$
G(s)=\frac{\left(K_{p}+K_{i} / s+K_{d} s\right) m e^{-s T}}{s^{2}-k^{2}}
$$

There are poles at $s=0$ and at $s= \pm k$; so the Nyquist contour must bypass the origin with a semicircle of zero radius as shown in Fig. 4. Then, $P=1$ and $N$ must equal -1 for stability. This means that the contour in the $G(s)$ plane must encircle $(-1,0 j)$ once in the anticlockwise direction. The Nyquist diagram may take any of the forms shown in Fig. 5, depending on the relationships between the controller parameters and the time delay.

If $T=0$ and $q<-1$ then $N=-1$ and the system is stable [Fig. 5(a)]. This corresponds to the requirement that the magnitude of $G(j \omega)$ is greater than unity when the phase lag is $180^{\circ}$. The angle requirement corresponds to $\omega^{2}=K_{i} / K_{d}$ which, when substituted into the magnitude requirement gives the following condition for stability:

$$
K_{p}>\frac{k^{2}}{m}+\frac{K_{i}}{K_{d} m}
$$

This shows that when the time delay $T$ is zero stable operation can be obtained using only proportional control provided $K_{p}$ is sufficiently large.

In Fig. 5(b) the controller settings are such that $N=1,3,5, \ldots$ depending on how many times the spiral encircles $(-1,0 j)$ and so the system is always unstable. In Fig $5(\mathrm{c}), N=-1$ and the system is stable if $q_{1}<-1<q_{2}$ but otherwise is not stable. The criterion for stability may be stated by requiring that the phase lag be less than $180^{\circ}$ when the magnitude of $G(j \omega)$ is unity, although this must be applied 

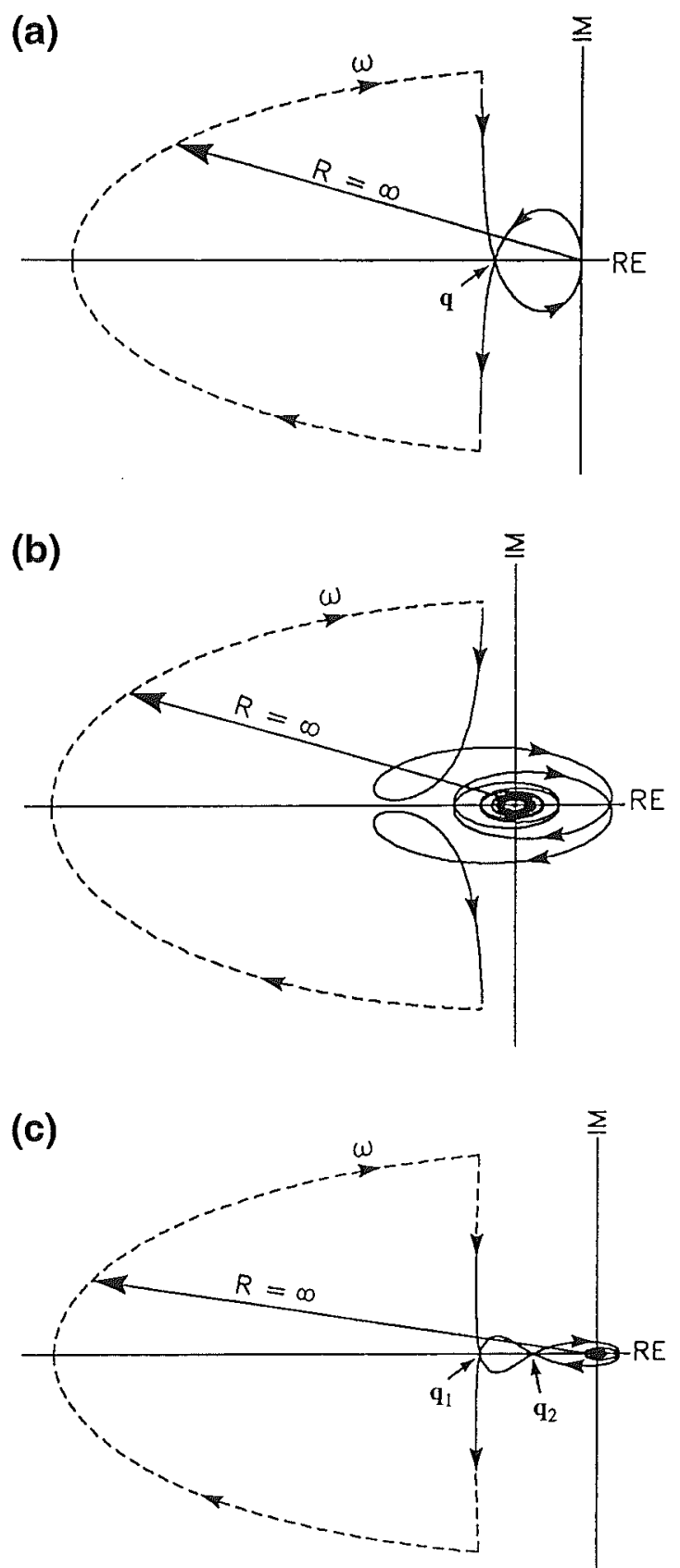

Figure 5: Nyquist diagrams for PID control with various values of the control parameters and the time delay $T$. In (a) $T=0$ and control is stable. In (b) $T>0$ and the system is unstable. In (c) $T>0$ and control is stable providing $q_{1}<-1<q_{2}$. 
with care because there may be more than one frequency at which the magnitude is unity. In equation form the requirements are:

$$
\omega T<\tan ^{-1}\left[\frac{\omega K_{p}}{K_{i}-K_{d} \omega^{2}}\right]-\frac{\pi}{2}
$$

when

$$
\left[\left(K_{i}-K_{d} \omega^{2}\right)^{2}+\left(K_{p} \omega\right)^{2}\right]^{1 / 2}=\omega\left(\omega^{2}+k^{2}\right) / m
$$

where $\omega$ is the frequency at which $|G(j \omega)|=1$. It is necessary that the inverse tangent term in equation (16) be greater than $\pi / 2$ for any $T$ and so:

$$
\omega^{2}>K_{i} / K_{d}
$$

Also since the inverse tangent term cannot exceed $\pi$, the maximum $T$ beyond which stable control is not possible is given by:

$$
T<\frac{\pi}{2 \omega}
$$

where $\omega$ is determined by the controller's parameters as described by equation (17). Equation (19) indicates that $\omega$ should be small if $T$ is large and hence from equation (18) $K_{i}$ should be small and $K_{d}$ should be large. Making $K_{i}$ small relative to $K_{d}$ implies that PD control should be used rather than PID control for dealing with time delays. In other words, the arm angular velocity $\dot{\varepsilon}$ should be based on the twist angle $\psi$ and the twist angular velocity $\dot{\psi}$.

Setting $K_{i}=0$ in equations (15)-(17) gives:

$$
\begin{gathered}
K_{p}>k^{2} / m \\
k T<\frac{\tan ^{-1}\left(\omega K_{d} / K_{p}\right)}{(\omega / k)}
\end{gathered}
$$

where

$$
\frac{\omega}{k}=\left[\frac{K_{p} m}{k^{2}} \sqrt{1+\left(\omega K_{d} / K_{p}\right)^{2}}-1\right]^{1 / 2}
$$

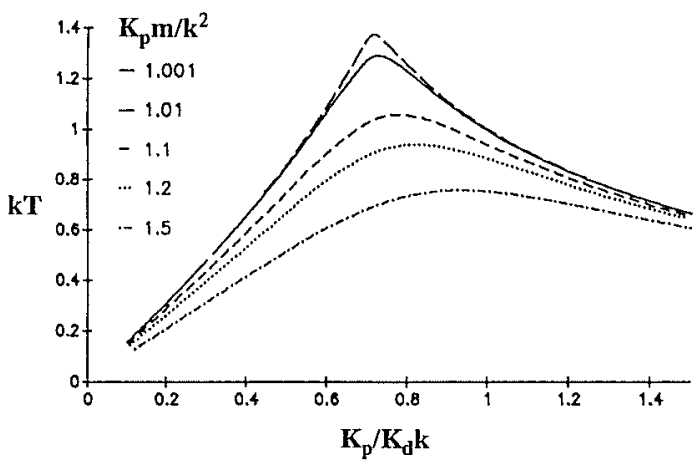

Figure 6: The time delay $T$ that can be accommodated for PD parameters $K_{p}$ and $K_{d}$. There are values of $K_{p}$ and $K_{d}$ which maximise $T$. For delays greater than this value of $\mathrm{T}$, stable control is not possible.

This shows $k T$ has a limiting value which depends that on $K_{p} m / k^{2}$ and $\omega K_{d} / K_{p}$. For the case of proportional control (21) shows that $T$ must be zero and so stable control is not possible using only proportional control when there is a finite time delay. For a fixed value of $K_{p} m / k^{2}$ (which must be greater than unity), each value of $\omega K_{d} / K_{p}$ has a corresponding $\omega / k$ and hencea corresponding $K_{p} / K_{d} k$. This enables $k T$ to be plotted against $K_{p} / K_{d} k$ for each value of $K_{p} m / k^{2}$ as shown in Fig. 6. Thus there is a 
set of controller values which maximises the time delay that can be accommodated. Alternatively, it may be stated that for each set of controller values, there is a value of time delay beyond which stable control is not possible. The larger the time delay, the narrower the range of allowable controller parameters and therefore greater the difficulty in maintaining stable motion. The maximum controllable time delay can also be estimated using the Routh-Hurwitz stability criterion with a second order approximation for the time delay. This gives a value of $k T=\sqrt{2}$, which is in close agreement with the result shown in Fig. 6 as $K_{p} m / k^{2}$ approaches unity.

\section{PD control}

The results of the numerical solutions for control using the computer simulation model were in general agreement with the theoretical findings. Proportional plus derivative controllers were used in simulations with perpendicular arms and with $0.1^{\circ}, 1^{\circ}$ and $10^{\circ}$ of initial arm asymmetry. A range of proportional and derivative parameter values was used in order to determine values which resulted in stable control. Increasing the time delay resulted in ranges of suitable parameter values and this enabled optimum values to be determined. For a time delay equivalent to 0.02 somersaults and an initial arm asymmetry of $10^{\circ}$, the arms moved rapidly to approximately symmetrical positions which changed little during the movement so that the response was stable [Fig. [7(a)]. When the delay was increased to 0.12 somersaults the twist was controlled although the amplitude of the arm oscillations did not decrease [Fig. 7(b)] and the response could be described as neutral control. Increasing the delay to 0.24 somersaults resulted in control of the twist although the difference in arm abduction angles became as much as $100^{\circ}$ [Fig. 7(c)] and the response was unstable.

If the original asymmetry in arm angles is reduced from $10^{\circ}$ to $1^{\circ}$ then a delay of 0.24 somersaults produces neutral response similar to Fig. 7(b). A further reduction in arm angle asymmetry to $0.1^{\circ}$ with a delay of 0.24 somersaults produces stable response similar to Fig. 77(a). Since the theoretical analysis of PD control gave a limit on the delay equivalent to 0.28 somersaults, the results of the numerical simulation model may be considered be to comparable. The best values for the proportional and derivative constants $K_{p}$ and $K_{d}$ were found by running numerous simulations. The value obtained for $K_{p} m / k^{2}$ was 1.01 which agrees well with Fig. 6] where this parameter should be slightly greater than 1.0 in order to maximise the time delay that can be handled. For a value of 1.01 Fig. 6 indicates a maximum time delay equivalent to 0.26 somersaults for which stable control can be maintained. The value of $K_{d}$ obtained empirically for the numerical simulations was equal to 0.98 of the optimum value indicated in Fig. 6] It may be concluded that there is good agreement between analytical and numerical results.

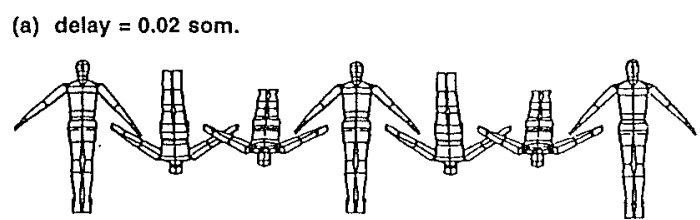

(b) delay $=0.12$ som.

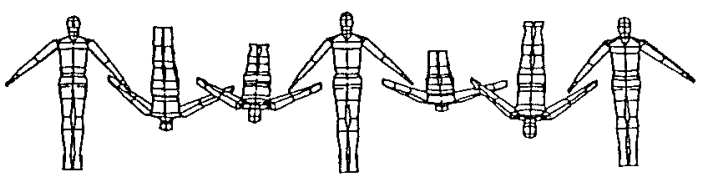

(c) delay $=0.24$ som

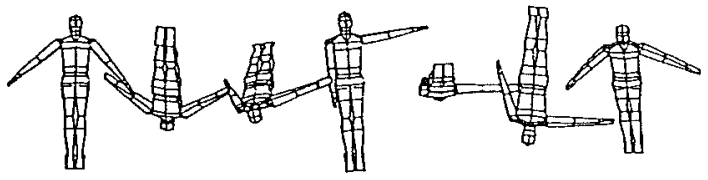

Figure 7: Proportional plus derivative control in double straight somersaults with feedback delays of (a) 0.02 , (b) 0.12 and (c) 0.24 somersaults. 
(a) film

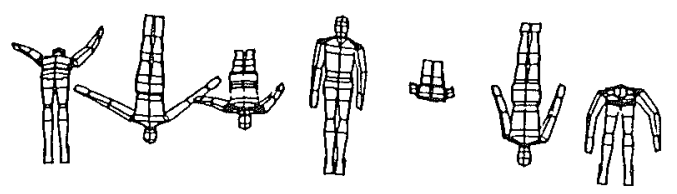

(b) simulation

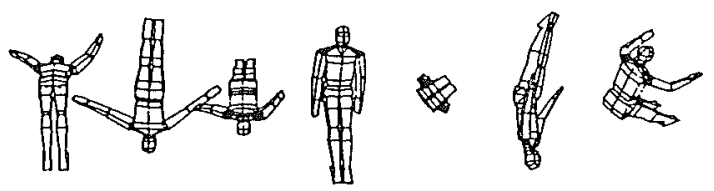

(c) simulation with control

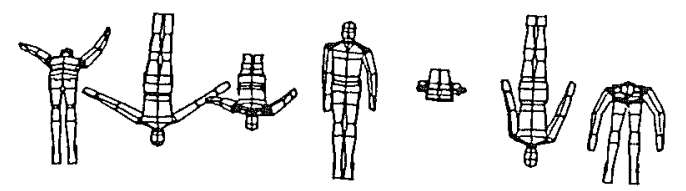

Figure 8: Performance of a double layout somersault obtained using (a) film analysis, (b) simulation and (c) simulation with control.

\section{Film analysis}

The three-dimensional film analysis of a double backward layout somersault performed by the elite trampolinist [Fig. 8(a)] revealed that the lateral axis through the hips was close to the intermediate principal axis for almost the entire movement. This suggests control that was employed during flight in order to prevent the build up of twist although this is not necessarily so (Table 2).

In order to determine whether there was sufficient body flexion to prevent a noticeable build up of twist, the film values of the internal orientation angles used to specify body configuration together with the initial whole body orientation and the angular momentum calculated as in Yeadon (1990c) were used as input to a computer simulation model (Yeadon et al., 1990). The agreement between film and simulation was good during the first somersault [Fig. 8(a) and (b)]. During the second somersault the effects due to instability became pronounced and the twist angle exceeded $90^{\circ}$ in the simulated movement. This indicates that without correction the instability would have led to noticeable twist after two somersaults. The discrepancy between simulation and film is to be expected since the error in the configuration angles obtained from film is around $1^{\circ}$ and this is sufficient to produce substantial twist in the second somersault (Table 10).

Control was introduced into the simulation by allowing the arm abduction angles to deviate from the film values by up to a maximum of $5^{\circ}$. The result was that the twist was controlled and the agreement with the film sequence became good throughout the simulation [Fig. 8(c)]. If more than two of the 14 configuration angles are used to control the twist, the constraint imposed the on difference between each film and control angle can be reduced from the $5^{\circ}$ used here.

\section{Visual feedback}

The experiment using visual deprivation of a trampolinist showed that it was not necessary to have visual feedback in order to maintain control in a double layout somersault. The trampolinist first performed six double layout somersaults from a plain jump. He was then instructed to close his eyes immediately after takeoff and to open them when instructed later in the flight phase. In the first two attempts at double layout somersaults under these conditions the trampolinist completed one and a half somersaults without twist before being instructed to open his eyes. During the last half somersault a quarter twist occurred on both occasions. The trampolinist reported that he was aware of the instability but was uncertain about trying to correct it with his eyes closed. He was instructed to make adjustments with his eyes 
closed. In the next four attempts the trampolinist successfully completed a double layout somersault with eyes closed for the first one and a half somersaults.

\section{DISCUSSION}

In this study the abilities of various hypothetical strategies for controlling twist in non-twisting somersaults have been evaluated using computer simulations. Such theoretical analyses cannot indicate which techniques are actually used by gymnasts but can indicate whether a proposed technique is viable. The results of this study should provide a useful starting point for investigations on the techniques actually employed by competitive athletes.

The strategy of symmetrically abducting the arms during flight, as advocated by Nigg (1974), has been shown to be incapable of reducing the build up of twist in layout double somersaults (Table 1). Flexing the body during flight progressively reduces the build up of twist for small perturbations even when there is sufficient flexion to ensure that the motion is in the stable wobbling somersault mode (Table 2). In a single somersault it may not be necessary to make corrections [Fig. 8(b)]. This contradicts the speculation of Hinrichs (1978) for somersaults about the unstable intermediate axis. For larger perturbations flexion sufficient to ensure the motion is in the stable mode is not sufficient to ensure that there is no appreciable build up of twist (Table 2). In this case additional flexion is required to limit the magnitude of the twist. Although it is possible to control the instability in a double layout somersault using body flexion, the competitive athlete is likely to lose points due to form breaks (Fig. 3) since it is an expectation of judges that the body will appear to be straight.

It has been shown that the build up of twist can be controlled using appropriate arm movements providing that the feedback time delay is not greater than a quarter of a somersault. For double layout somersaults in a gymnastics floor exercise this delay is equivalent to about $150 \mathrm{~ms}$ which cannot be much more than a gymnast's reaction time. In this theoretical study the angular velocity $\dot{\varepsilon}$ of the arm movement was used as the control variable and was a linear function of the twist angle $\psi$ and twist angular velocity $\dot{\psi}$ for PD control. In a practical situation, control will be effected using neural stimulation of the appropriate muscle groups and this input will be related to the joint torques. This suggests that the control variable used by gymnasts will be similar to the angular acceleration $\ddot{\varepsilon}$ which would be a function of $\dot{\psi}, \psi$ and $\ddot{\psi}$ for PID control. Thus the result that PD rather than PID control should be used is equivalent to saying that gymnasts must base their control on the twist angular velocity and acceleration values but not on the twist angle itself. Since the otolith organs of the inner ear can detect angular velocities due to centrifugal effects and the semicircular canals respond to rotational accelerations (Wendt, 1951), it is possible that vestibular control is used rather than visual control. This idea is supported by the experiment involving closing the eyes during a double layout somersault. The main function of the eyes may be to obtain angular information on body orientation in space in order to make in-flight adjustments for correct landing orientation rather than to control instability during flight (Rezette and Amblard, 1985).

Playter and Railbert (1994) showed that a three segment model, comprising a body and two arms with torsional springs at the shoulders, automatically makes corrective movements when somersaulting. Providing that the arms are abducted more than about $30^{\circ}$ from the mid-line of the body and that the springs have suitable stiffness, the system is neutrally stable and will perform double and triple somersaults without appreciable twist. The implication of this study of passive stability is that the bodies of gymnasts may automatically and instantaneously make compensatory movements. The majority of double layout somersaults are performed with the arms adducted close to the body. In this configuration passive corrections are insufficient to prevent the build up of twist whereas the simulation using PD control based on film data [Fig. 8(c)] shows that active corrections can maintain control. This indicates that passive corrections are not of mechanical importance in the control of twist. On the other hand, the tendency of the limbs to move in the appropriate direction may provide additional feedback information for input to the control system used.

\section{REFERENCES}

Hinrichs, R. N. (1978) Principal axes and moments of inertia of the human body: an investigation of the stability of rotary motions. Unpublished MA thesis. University of Iowa. 
Marion, J. B. (1965) Classical Dynamics of Particles and Systems. Academic Press, New York.

Nigg B. M. (1974) Analysis of twisting and turning movements, In Biomechanics IV, (Edited by Nelson, R. C. and Morehouse, C. A.), pp. 279-283. Macmillan, London.

Playter, R. and Railbert, M. (1994) Passively stable layout somersaults, In Canadian Society for Biomechanics: Proc. Eighth Biennial Conf. and Symp., pp. 158-159. University of Calgary.

Rezette, D. and Amblard, B. (1985) Orientation versus motion visual cues to control sensorimotor skills in some acrobatic leaps. Hum. Mvmt. Sci. 4, 297-306.

Van de Vegte, J. (1986) Feedback Control Systems. Prentice-Hall, Englewood Cliffs, NJ.

Wendt, G. R. (1951) Vestibular functions, In Handbook of Experimental Psychology (Edited by Stevens, S. S.), pp. 1191-1223. Wiley, New York.

Yeadon, M. R. (1989) A method for obtaining three-dimensional data on ski jumping using pan and tilt cameras. Int. J. Sport Biomech. 5, 238-247.

Yeadon, M. R. (1990a) The simulation of aerial movement - I: The determination of orientation data. J. Biomechanics 23, 59-66.

Yeadon, M. R. (1990b) The simulation of aerial movement - II: A mathematical inertia model of J. Biomechanics 23, 67-74.

Yeadon, M. R. (1990c) The simulation of aerial movement - III: The determination of the angular momentum of the human body. J. Biomechanics 23, 75-83.

Yeadon, M. R., Atha, J. and Hales, F. D. (1990) The simulation of aerial movement - IV: A computer simulation model. J. Biomechanics 23, 85-89.

Yeadon, M. R. (1993a) The biomechanics of twisting somersaults. Part I: Rigid body motions. J. Sports Sci. 11, 187-198.

Yeadon, M. R. (1993b) The biomechanics of twisting somersaults. Part III: Aerial twist. J. Sports Sci. 11, 209-218. 